\title{
ENGINEERING DEVELOPMENT OF PASSIVE IONOSONDE TO STUDY THE EFFECT OF THE BLACKOUT ON HF COMMUNICATION
}

\author{
Ruslan Belgibaev* \\ Volga State University of Technology, Yoshkar-Ola, Russia \\ Dmitriy Ivanov \\ Volga State University of Technology, Yoshkar-Ola, Russia \\ Vladimir Ivanov \\ Volga State University of Technology, Yoshkar-Ola, Russia \\ Natalya Ryabova \\ Volga State University of Technology, Yoshkar-Ola, Russia \\ Maria Ryabova \\ Volga State University of Technology, Yoshkar-Ola, Russia
}

The development of passive ionosonde that enables to assess the information of technological characteristics of ionospheric radio channel in real time, was carried, which is part of the long distance HF communications systems. On its basis there was conducted applied research of the effect of radio blackout of HF communications caused by the $X$-ray flare on the sun on the frequency capacity of the multi-dimensional ionospheric radio. It was found out that the blackout of R2 category leads to the cessation of HF communication about 2600 telephone channels, i.e. frequency channel capacity is reduced by $34 \%$. It was set that the relaxation time of blackout is in 1.7 times more than the relaxation time of the $X$-ray flash.

Key words: HF communications, Passive Probe, Blackout, RF radio frequency capacity

\section{INTRODUCTION}

Short waves (high frequency - HF) are an effective mean of solving the problem of providing wireless communication over long distances and communications in areas with difficult lay of the land (mountains). These unique properties of HF are due to the effect of high-frequency reflection from the Earth's ionosphere. In this case, the propagation medium is identified with the radio channel. On the ionospheric HF communication lines it is possible to organize $\mathrm{J}$ partial adjacent frequency channels. Everyone can have different settings. The main ones are: signal/noise ratio and scattering in the time and frequency domains. The whole set of partial channels can be considered as a multi-dimensional radio dimension [01, 02]. Clearly, the dimension $\mathrm{J}$ of the channel matches with frequency capacitance of ionospheric line RF communication.

The main problems that negatively affects the operation of the HF communication [03-09] are unpredictable perturbations of dimension and parameters of the multidimensional radio channel. All of these, including dimension is actually a function of time. Physical processes that lead to such changes, are solar flares, the disturbance of the magnetic field of the Earth, the sporadic E layer, and others. In this regard, we have the problem of determining of the current the frequency capacity of the link and select of optimal from the $\mathrm{J}$ channels for its implementation. These problems are solved by periodically sensing ionospheric link (multidimensional channels).

Nowadays two types of ionosondes we are used for probing: panoramic and channel. Mostly used are panoramic ionosondes with continuous linear frequency-modulated (LFM) signal. For them, the optimal treatment is effective probing signal in the receiver, which allows you to get a significant (up to ) gain in the value of the signal/ noise ratio and significantly reduce the power of the probe signal. However, for the implementation of the chirp lonosonde requires expensive special equipment. 
For multi-dimensional sensing radio, components of which are adjacent partial narrowband channels may use a passive chirp ionosonde. In this case, for receiving sounding signals can use the communications equipment. However, the approach of passive sensing panorama demands the development of algorithmic and software parts. This is particularly important for the study of the influence of the ionospheric disturbances on the multi-dimensional radio channel. One of them is a little-studied effect of radio blackout caused by X-ray solar flares and the related sudden decrease in the frequency capacity link.

The aim of the work is the creation of algorithms and software for the development of a method of passive sensing panoramic multidimensional radio and research based on them the effect of radio blackout.

\section{DEVELOPMENT OF A PASSIVE PROBE FOR THE DIAGNOSIS OF HF RADIO MULTIDIMENSIONAL}

To solve this problem questions of a theoretical nature were investigated. It is taken into account that the transmitters emit a continuous panoramic ionosondes chirp signal in the band of $3-30$ $\mathrm{MHz}$ at a speed adjustment $50-500 \mathrm{kHz} / \mathrm{sec}$. Suppose that the receiver is spaced from the transmitter and set the operating frequency $\bar{f}$. If the bandwidth of the receiver is equal to $\mathrm{Bc}$, then it cuts out from a continuous chirp signal pulse with $T_{0}=1 / B_{c}$.

Further adopted chirped pulse is digitized, smoothed by Gaussian-like Hamming window and exposed "deciphering" in the time domain. It is known [17] that a truncated Gaussian chirp signal by the level of $e^{-2}$ at the points $t= \pm T_{0} / 2$ due to the passage of the filter strip $B_{c}=\dot{f} \cdot T_{0}$ is as follows:

$$
\begin{aligned}
& \left.u(t)=u_{0} \cdot \exp \mid-4(t / T)^{2}\right] \cdot \exp \left[\beta\left(t-t_{0}\right)^{2} / 2\right]= \\
& =u_{0} \cdot \exp \left[-8\left(t / T_{0}\right)^{2}\right] \cdot \exp \left[\beta\left(t-t_{0}\right)^{2} / 2\right]= \\
& =u_{0} \cdot \exp \left[-8 \dot{f}^{2}\left(t / B_{c}\right)^{2}\right] \cdot \exp \left[\beta\left(t-t_{0}\right)^{2} / 2\right]
\end{aligned}
$$

where $T=T_{0} / \sqrt{2}$ - the duration of the chirped pulse level $e^{-1}, \beta=2 \pi \dot{f}$ and $t_{0}$ - chirp-time (chirptime), the time for which the frequency of the emitted chirp signal would be zero,because $\omega=\beta\left(t-t_{0}\right)$.

We assume that when compressed signal amplitudes of all harmonics (1) shall be transferred without changes, and the phase response of the filter matched to the phase of its spectrum. Then as a result of multiplication in the frequency domain spectrums obtain:

$U_{R}(\omega)=u_{0} \sqrt{\frac{\pi}{\beta}} \cdot \exp \left[-8\left(\omega / \Omega_{c}\right)^{2}\right]$,

The compressed time-domain chirp pulse will coincide with the inverse Fourier transform of the spectrum (2). As a result, we have [18]:

$|U(\tau)|=u_{0} \sqrt{m} \exp \left[-\left(\frac{\tau}{\mathrm{T} / m}\right)^{2}\right]=u_{0} \frac{\sqrt{\pi B_{c} T_{0}}}{2 \sqrt{2}} \exp \left[-\left(\frac{\pi B_{c} \tau}{2 \sqrt{2}}\right)^{2}\right]$,

where $\mathrm{T}=T_{0} / 2 \sqrt{2}, m=\Omega_{c} T_{0} / 16$.

Width of compressed Gaussian pulse at $3 \mathrm{~dB}$ equals $1.5 / \mathrm{B}_{\mathrm{c}}$. Estimates of the compressed pulse width of the square give the value of $0.9 / B_{c}$, and pulse with an envelope Hamming $-1,3 / B_{c}$.

From the expression (3) for instant power delay profile of channel on frequency $\bar{\omega}$, taking into account the delay and multipath reception, but neglecting the interference, we obtain:

$P_{\tau}(\bar{\omega}, \tau)=|U(\tau)|^{2}=\frac{\pi B_{c} T_{0}}{8} \sum_{n=1}^{N} u_{0_{n}}^{2} \exp \left[-\frac{1}{2}\left(\pi B_{c}\left(\tau-\tau_{n}\right)\right)^{2}\right]$

Its width is in 1.4 times less than that of the envelope of the compressed pulse, however, it is still at such a channel band power delay profile of received mode taken not permitted delay.

Consistent sounding radio with different average frequency allows to define the power delay profile of multidimensional channel in the frequency range [LUR, MUF]:

$\vec{P}_{\tau}(\bar{\omega}, \tau, t)=\left\{P_{\tau}\left(\bar{\omega}_{1}, \tau, t\right)\right.$;

$\left.P_{\tau}\left(\bar{\omega}_{2}, \tau, t\right) ; \ldots ; P_{\tau}\left(\bar{\omega}_{J}, \tau, t\right)\right\}$.

Bitmap of power delay profile provides a "portrait" of a multidimensional channel and allows to determine the lowest usable frequency (LUF) and the maximum usable frequency (MUF) line of HF communication.

\section{THE STRUCTURE OF PASSIVE CHIRP IONOSONDE}

A block diagram of an apparatus for passive sounding of multidimensional HF radio channel is shown in Figure 1.

Chirp lonosonde operation known in place of reception and the receiver of passive probe is incrementally rebuilt after changes the frequency of the emitted signal. Tuning step is $100 \mathrm{kHz}$ and the band of the receiver (bandpass filter frequency channel) is $2.4 \mathrm{kHz}$. 


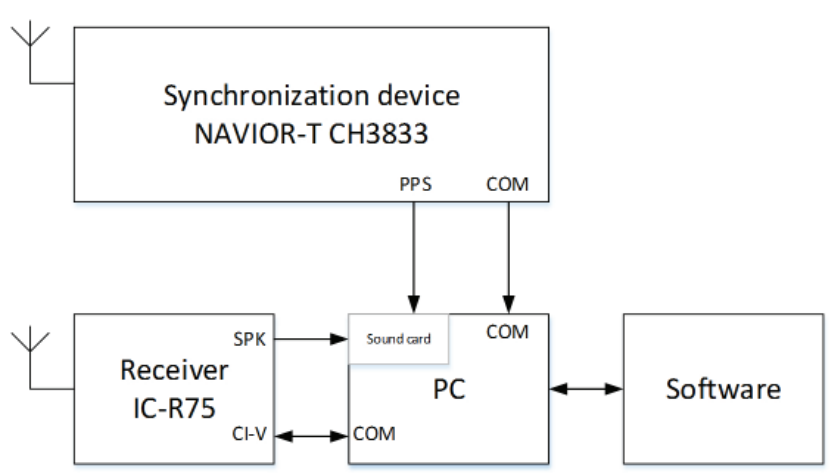

Figure 1: Block diagram of the device for passive sensing multidimensional ionospheric HF radio

Restructuring the receiver by means of the control unit is synchronized in time and central frequency of the channel. Receiver passive probe "cuts" in the received signal continuously chirped pulse with a deviation equal to the bandwidth of the receiver, and the duration $\mathrm{T}_{0}=24 \mathrm{~ms}$. The compressed time-domain chirp pulse was $0.54 \mathrm{~ms}$.

Time-frequency synchronization [14] is carried out by the signals of navigation systems GLONASS and GPS by the module NAVIOR-T SN3833. The received low-frequency chirp signal is converted to digital using the sound card and its coherent processing, determination of the parameters of the partial channels and the mapping information is performed in a personal computer $(\mathrm{PC})$ using the signal processing module. General view of the passive chirp lonosonde created in VSUT is shown in Figure 2.

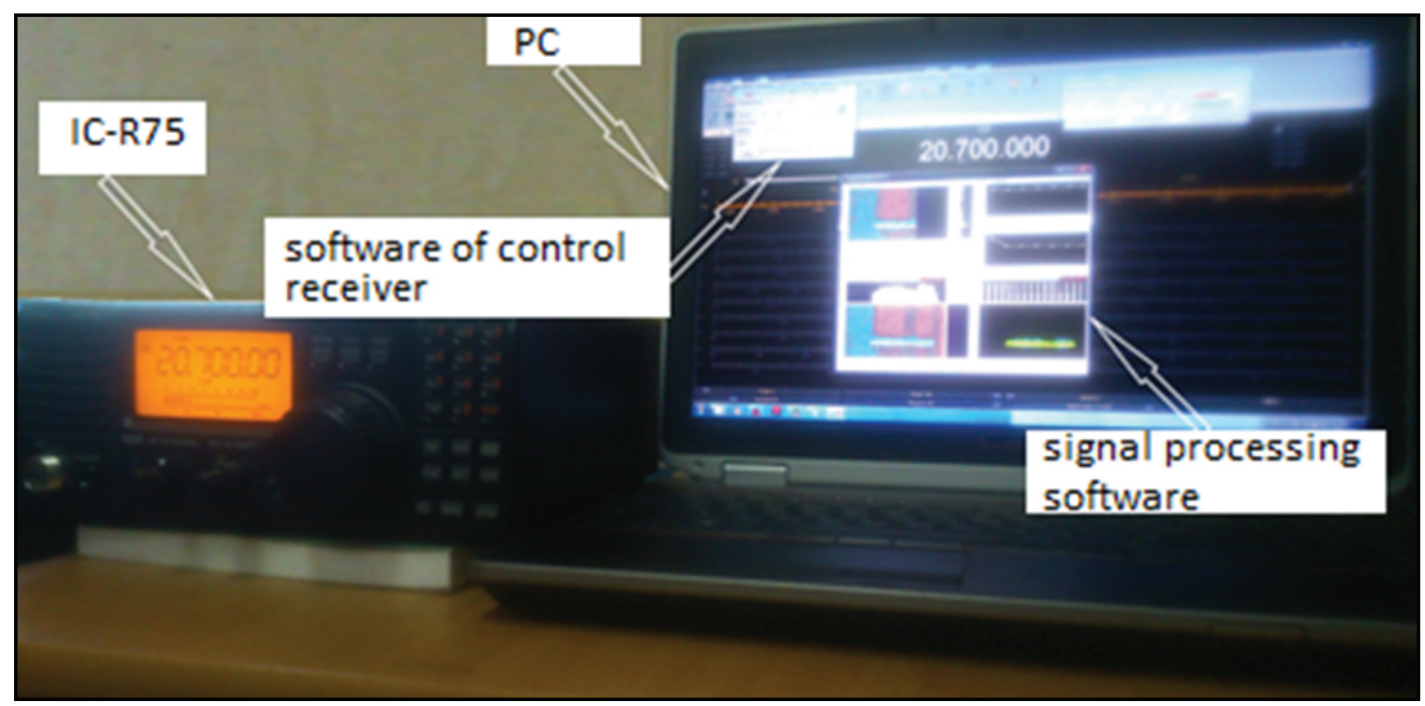

Figure 2: Passive chirp lonosonde

In the figure 3a as an example of the partial power delay profile of channel marked on Figure $3 b$ white vertical stripe. Figures 3b, c.- power delay

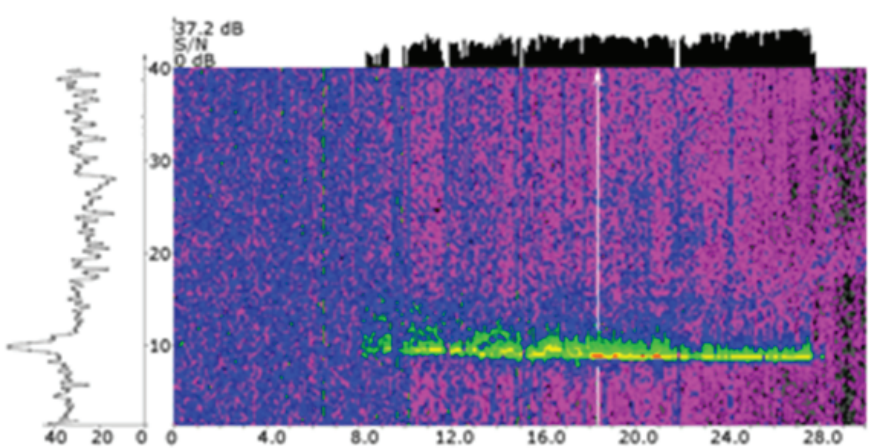

(a) profile of multidimensional channel for the same radio Cyprus - Yoshkar-Ola length of $2630 \mathrm{~km}$. The color in the figure shown signal strength.

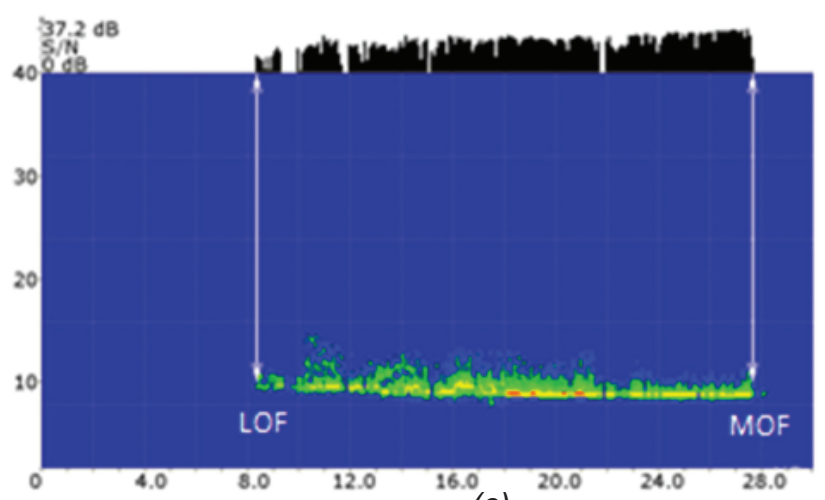

(c)

Figure 3: The output signal of the matched filter (a) power delay profile of multidimensional HF radio channel for the path of Cyprus - Yoshkar-Ola (b) and power delay profile cleared of noise (c)

Figure $3 \mathrm{c}$ is characterized in that it removed the image processing methods the noise component. The first range on power delay profile take for the lowest usable frequency (LUF) and the last - of the maximum usable frequency (MUF) (see. Figure 4c). Algorithms for detecting and isolating the wanted signal from noise, given in $[15,16]$. 


\section{THE DIMENSION OF THE IONOSPHERIC HF RADIO}

By the propagation in ionosphere amplitudephase relationships of signal are broken, which leads to a distortion of the received signal. There are several approaches to describing the radio propagation environments. However, most developed an approach where the physical propagation medium is replaced by an equivalent linear system [03, 07,10-13]. This problem is most easily solved when the problem of propagation of radio waves seen in the ray approximation. In this case, every incoming beam to the receiver can associate a path equivalent linear system.

Usually the linear is system described by system characteristics: frequency response (frequency response) of the linear system (radio), which determines the response of the system to any input action (signal) and the impulse response (s):

$$
H(\omega)=R_{0}(\omega) \cdot \exp \left(-j \int k d r\right)
$$

and

$$
h(\tau)=\frac{1}{2 \pi} \int_{-\infty}^{\infty} H(\omega) \cdot \exp (j \omega \tau) d \omega
$$

It is known that high-frequency electromagnetic oscillations range from transmitter to receiver, separated by thousands of kilometers, reaching the radio waves at frequencies $f_{m}=L U F$ to $f_{M}=M U F$. Therefore ionospheric link can be arranged $\mathrm{J}$ with radio frequency band of $B_{c}$, where $J=\left(f_{M}-f_{m}\right) / B C$. Therefore, the parameter $\mathrm{J}$ can be considered as the frequency of HF communication line capacity.

Considering formula (6) for IR channel can obtain the following expression:

$$
\begin{aligned}
& h(\bar{\omega}, \tau, t)=\frac{1}{2 \pi} \int_{-\infty}^{\infty} H(\omega, t) \cdot \exp (j \omega \tau) d \omega= \\
& =\frac{1}{2 \pi} \int_{\omega_{m}}^{\omega_{1}}+\frac{1}{2 \pi} \int_{\omega_{1}}^{\omega_{2}}+\ldots+\frac{1}{2 \pi} \int_{\omega_{M-1}}^{\omega_{M}}= \\
& =h\left(\bar{\omega}_{1}, \tau, t\right)+h\left(\bar{\omega}_{2}, \tau, t\right)+\ldots+h\left(\bar{\omega}_{J}, \tau, t\right)
\end{aligned}
$$

where $\bar{\omega}_{J}=\bar{\omega}_{1}+J \cdot B_{c}, h\left(\bar{\omega}_{J}, \tau, t\right)$ - partial channel. Equation (7) allows us to consider the whole set of partial channels HF link as they are multi-dimensional (vector) HF radio coordinates:

$$
\begin{aligned}
& \vec{h}(\bar{\omega}, \tau, t)=\left\{h\left(\bar{\omega}_{1}, \tau, t\right) ;\right. \\
& \left.h\left(\bar{\omega}_{2}, \tau, t\right) ; \ldots ; h\left(\bar{\omega}_{J}, \tau, t\right)\right\} .
\end{aligned}
$$

The dimension of the channel coincides with the multidimensional frequency capacity of HF link. The study of the influence of sudden ionospheric disturbances on frequency capacitance (the dimension of the channel) link is one of the objectives of our work.

\section{TYPES OF IONOSPHERIC DISTURBANCES RADIO BLACKOUTS}

Whereas in the unperturbed ionosphere is a complex and very heterogeneous system, there are mechanisms that can cause serious deviations of its parameters, collectively known as ionospheric disturbances. Disturbances of the ionosphere is a result of the events taking place on the sun. The main causes of ionospheric disturbances are solar flares. The X-ray flashes are separated by classes (see. Table 1).

Table 1: Classes of solar flares [19]

\begin{tabular}{|c|c|}
\hline Designation & $\begin{array}{c}\text { The intensity of the X-ray peak } \\
\left(\mathbf{W} / \mathbf{m}^{2}\right)\end{array}$ \\
\hline A & $<10^{-7}$ \\
\hline$B$ & $10^{-7}-10^{-6}$ \\
\hline C & $10^{-6}-10^{-5}$ \\
\hline M & $10^{-4}-10^{-5}$ \\
\hline X & $>10^{-4}$ \\
\hline
\end{tabular}

Effects and their underlying causes are shown in Figure 4. The effects caused by the events on the Sun, are divided into sudden ionospheric disturbances (SIDs), geomagnetic storms and polar cap absorption:

Sudden ionospheric disturbances (SIDs). Shortterm changes in the transparency of the radio spectrum, caused by the solar flare X-ray radiation, due to increased absorption in the $D$ - region. They have a fast rise (a few minutes) and a slower decline (usually within one or two hours). Sudden deviations frequencies cause the positive and negative Doppler changes in the propagation of radio signals, since the effective height of the layer initially decreases (due to increased ionization), and then returns to the initial value. If solar flares accompanied by a slight change in the absorption of the HF, the main cause SID is ultraviolet radiation.

Polar cap absorption (PCA). While large solar flares are emitted as high-energy protons. When entering the ionosphere, they cause ionization in collisions with gas molecules. The effect can last for several days and is manifested in the form 
of strong absorption of radio waves in the daytime. The effect begins at high latitudes and can spread to the south.

Ionospheric storm (IS). IS caused by exposure to a magnetic field of the Earth flows ejected in the outburst "magnetized" solar plasma. These include geomagnetic storms, auroras, etc., affect- ing the propagation of radio waves in the middle and low latitudes. IS may be accompanied by auroral absorption of radio waves in the $\mathrm{D}$ - region of auroral occurrence ES layer, increasing the thickness of $F_{2}$ layer, and an increase in the appearance of fading interference level. IS may take several days.

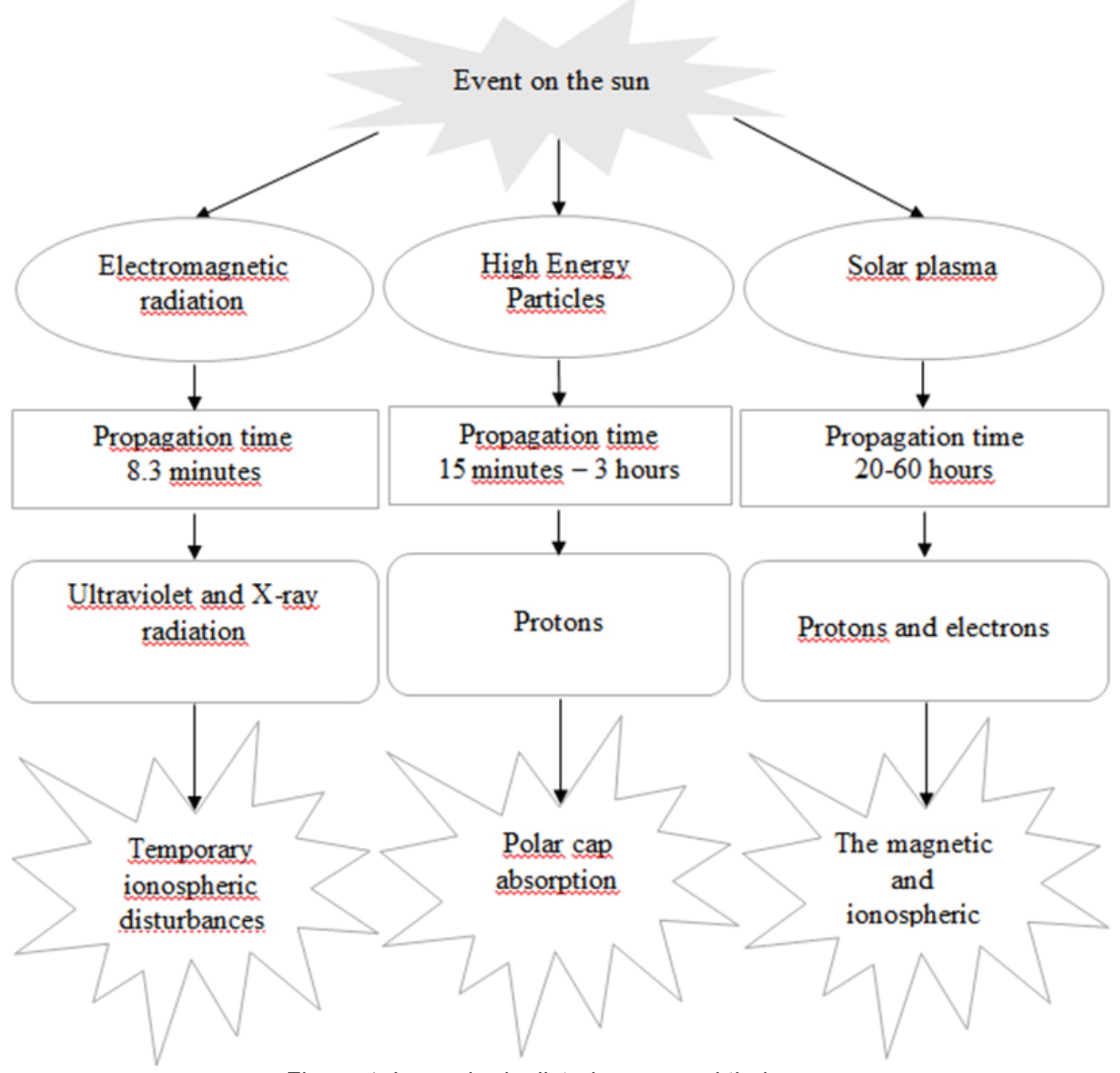

Figure 4: Ionospheric disturbances and their causes

The effect of absorption of radio waves at SID These events are difficult to predict, so it is necin the literature is often called a radio blackout. essary to conduct research and radiosonde to Blackout divided into classes (see. Table 2). register them.

Table 2: Classes of solar flares[20]

\begin{tabular}{|c|c|c|}
\hline Classification of NOAA & Class solar flares & Duration \\
\hline R1 & M1 & minutes \\
\hline R2 & M5 & tens of minutes \\
\hline R3 & X1 & about an hour \\
\hline R4 & X10 & $1-2$ hours \\
\hline R5 & X20 & few hours \\
\hline
\end{tabular}




\section{EXPERIMENTAL STUDY OF THE EFFECT BLACKOUT ON THE FREQUENCY CAPACITANCE MULTIDIMENSIONAL HF RADIO}

The developed algorithms and their implementation in the software part lonosonde held an experimental verification. The experiments were conducted in the period from 14 to 21 April 2014 midlatitude radio Cyprus - Yoshkar-Ola. April 18, 2014 there was an outbreak in the sun. This flash refers to the class M 7.3 (see. Figure 5a). Red shown the long wave flux of X-rays $\left(\lambda_{1}=\right.$ $0.1-0.8 \mathrm{~nm})$ and blue - short wave $\left(\lambda_{2}=0.05-0.4\right.$ $\mathrm{nm})$. During the outbreak of geomagnetic disturbances was low, as evidenced by the data presented in Figure $5 \mathrm{~b}$.

(a)

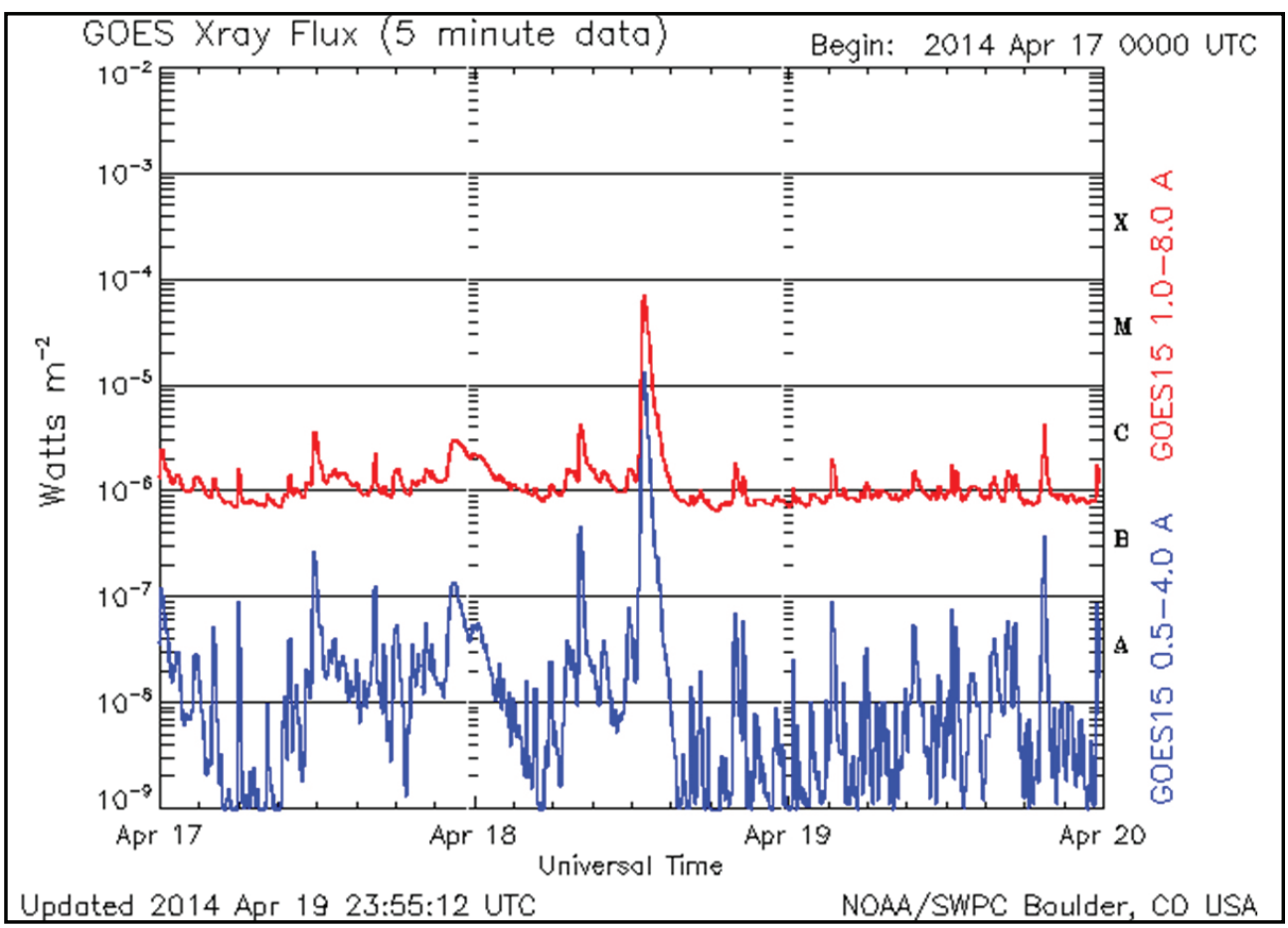

(b)

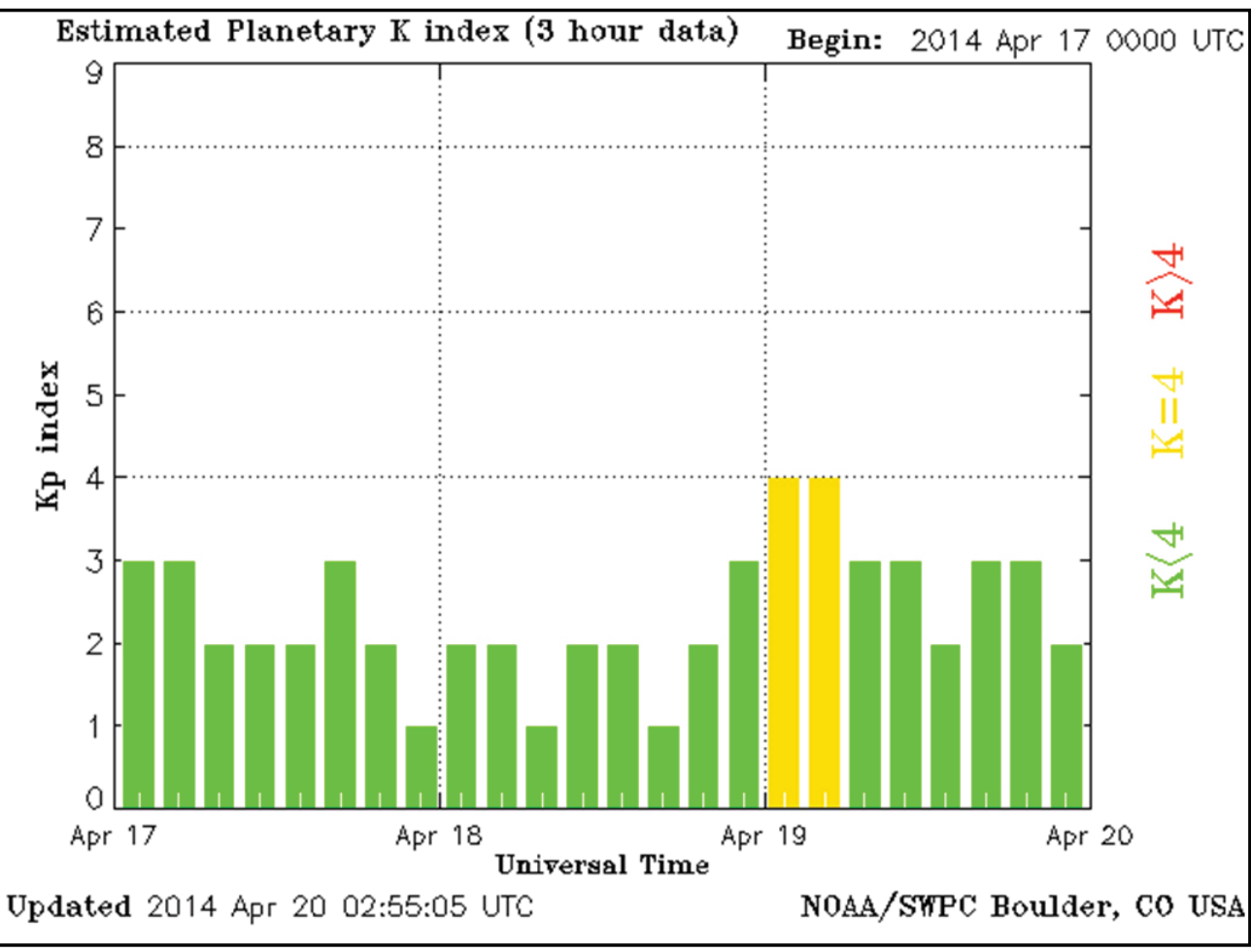

Figure 5: (a) X-ray flux density and (b) a planetary index Kp 
Using passive probe in the experiment power delay profile were received and they are presented in Figure 6 in the form of time sequence. It differs from Figure $4 b$ that the frequency axis is directed vertically and horizontally time delayed in increments of 5 minutes. Data on changes in the flux density of $X$-ray radiation obtained a geostationary satellite. Figure $6 \mathrm{~b}$ shows the course of a multi-dimensional channel frequency capacity and density of X-ray flux, normalized to unity.

Figure 6a contains information about the received signal power. We see now the X-ray intensity synchronous change of power of the radio medium with varying frequency throughout the frequency range of LUF to MUF (the color in the picture is shifted to the dark area) and the effect of blackout at frequencies below $16 \mathrm{MHz}$.

We see that the SID, leading to blackout affects the frequency capacity of the multi-dimensional channel (Figure 6b). If the bandwidth of the partial channel is $3 \mathrm{kHz}$, in the absence of disturbance on the radio Cyprus - Yoshkar-Ola could be organized $\sim 7660$ channels, full blackout led to their reduction to the value of $\sim 5056$. Thus, blackout category R2 reduces the frequency capacity of the line Communications Cyprus Yoshkar-Ola 34\% (about 2,600 channels).

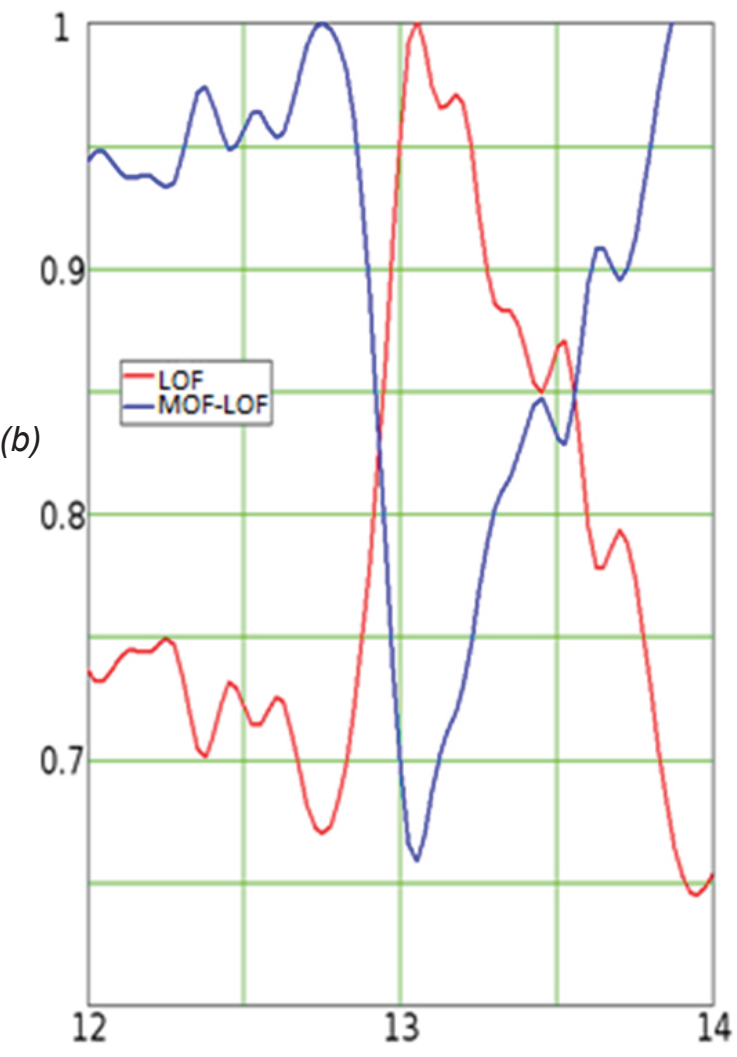

Figure 6: (a) Progress power delay profile multidimensional HF radio, $X$-ray flux with $\lambda_{1}=0.1-0.8 \mathrm{~nm}$ (blue) and $\lambda_{2}=0.05-0.4 \mathrm{~nm}$ (yellow) and (b) the relative frequency of the channel capacity of a multi-dimensional (blue color), the relative course of LUF (red)

Suppose that the relaxation of the events described by the law:

$y(t)=y_{m} \exp \left[-\frac{t-t_{m}}{T_{r}}\right]$

where $T_{r}$ - relaxation time.

Taking the logarithm of both sides of the expression (9), we obtain:

$z(t)=\ln \left(\frac{y(t)}{y_{m}}\right)=-\frac{t-t_{m}}{T_{r}}$

Journal of Applied Engineering Science 14(2016)4, 406
Note that the function $z(t)$ is linear with a slope equal to $1 / T_{r}$. Parameter defines a straight shift on the time axis.

To analyze the behavior of the event at the stage of relaxation all the experimental data were studied functions and logarithms samples were plotted on a graph (see. Figure 7), where the abscissa is time and the ordinate the logarithm of the ratio $\operatorname{Ln}\left[\mathrm{y}(\mathrm{t}) / \mathrm{y}_{\mathrm{m}}\right]$. As $y_{m}$ and $t_{m}$ were taken coordinates of highs functions. 


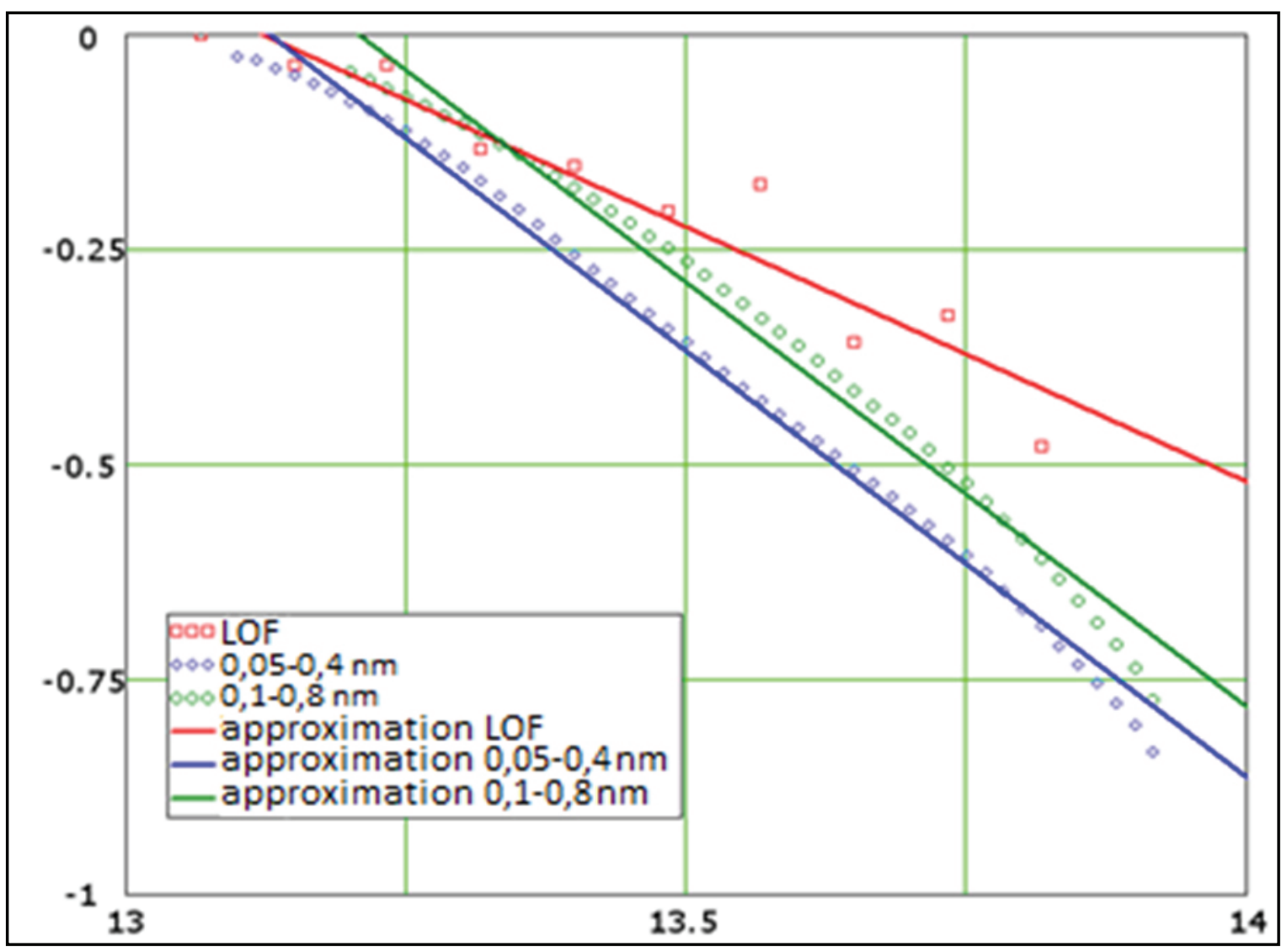

Figure 7: Relaxation stroke LUF (red dots), "soft" X-rays with $\lambda_{1}=0.1-0.8 \mathrm{~nm}$ (green dots) and "hard" with $\lambda_{2}=0.05-0.4 \mathrm{~nm}$ blue dots)

We see that the experimental points in the relaxation phase concentrated near the corresponding lines. This demonstrates the loyalty of our hypothesis. In addition, LUF monitor the progress of the intensity of "hard" X-rays, and the course of the "soft" X-rays is delayed relative to the time of $\sim 5$ minutes.

The parameters were obtained direct contact using the method of least squares. Carried there on relaxation time evaluation showed that it is respectively equal to:

$$
\left\{\begin{array}{c}
T_{R \lambda 1}=60.722[\mathrm{~min}] \\
T_{R \lambda 2}=60.537[\mathrm{~min}] \\
T_{R L U F}=101.46[\mathrm{~min}]
\end{array}\right.
$$

Note that the relaxation time of the "soft" and "hard" X-rays in 1.7 less than times relaxation LUF.

Since in our case, the frequency capacitance probed the link has a stroke reverse course LUF. This allows you to record the frequency capacitance expression of the following form:

$$
\frac{J(t)}{J_{\max }}=\exp \left(\frac{t-t_{m \lambda 2}}{1.7 \cdot T_{R \lambda 2}}\right)=\frac{\Pi_{\lambda 2}(t)}{\Pi_{\max } \lambda 2}
$$

Formula (12) to evaluate by the function act of increase the frequency capacity of the relaxation stage blackout on the relaxation flux of $\mathrm{X}$-rays.

\section{CONCLUSIONS}

1. The device for the diagnosis of HF ionospheric channel by passive sensing algorithms for optimal processing and visual display was developed.

2. As a result of using the device for diagnosing HF channel during blackout it was found out that blackout of R2 category reduces by $34 \%$ (2600 units) of its frequency capacitance. The relaxation time of blackout in 1.7 times more than the relaxation time of $\mathrm{X}$-ray radiation from the sun.

\section{ACKNOWLEDGEMENT}

This work was supported by grants RFBR project № 13-07-00371; 13-02-00524; 15-07-05280; 15-07-05294; Grants Ministry of Education of the Russian Federation № 3.2695.2014 / K, № 8.2697.2014 / K, № 2276, № 2247; RNF № 1519-10053. 


\section{REFERENCES}

1) Ivanov, D.V., Ivanov, V.A., Ryabova, N.V. and Chernov A.A., (2013) "Multidimensional highfrequency radio channel and experimental studies of its main characteristics", Electromagnetic Waves and Electronic Systems, 8(18), 40-48.

2) Ivanov, V.A., Ivanov, D.V., Ryabova, N.V. etc., (2011) "Determination of key parameters of a multidimensional short-wave radio channel with the use of a panoramic ionosonde", Vestnik of Volga State University of Technology. Series "Radio Engineering and Infocommunication Systems", 2, 15-23.

3) Kryukovsky, A.S. and Zaichickov, I.V., (2008) "Features of radiopulse propagation in media with dispersion", Electromagnetic Waves and Electronic Systems, 8(13), 36-41.

4) Ivanov, D.V., Ivanov, V.A. and Ryabova, N.V., (2010) "Sounding of the ionosphere and decametric communication channels by complex radio signals", Vestnik of Volga State University of Technology. Series "Radio Engineering and Infocommunication Systems", 1, 3-37.

5) Maslin, N.M., (1987) HF communications: a systems approach, Pitman Publishing, London.

6) Ivanov, V.A., Ivanov, D.V., Ryabova, N.V. and Tsarev, I. E., (2009) "Numerical and composite analysis's of narrow band HF-channels scattering function", Electromagnetic Waves and Electronic Systems, 8(14), 46-54.

7) Kryukovsky, A.S., Lukin, D.S. and Rastyagaev D.V., (2009) "Research of singularities of short radio wave propagation in non-uniform anisotropic ionosphere", Electromagnetic Waves and Electronic Systems, 8(14), 17-26.

8) Picket, (1985) "Real time tactical frequency management", IEEE Milit. Commun. Conference, Boston, 51-53.

9) Ivanov, D.V., Ivanov, V.A., Ryabova, N.V., Ryabova, M.I., Elsukov, A.A. and Chernov A.A., (2013) "SDR-ionosonde with continuous LFM-signal on the platform USRP", Vestnik of Volga State University of Technology. Series "Radio Engineering and Infocommunication Systems", 3(19), 80-93.

10) Erukhimov, L.M., Uryadov, V.P., Cherkashin, Yu.N., Eremenko, V.A., Ivanov, V.A., Ryabova, N.V. and Shumaev V.V., (1997) "Pedersen mode ducting in a randomly stratified ionosphere", Waves in Random Media, 4(7), 531-544.
11) Ivanov, V.A., Kurkin, V.I., Nosov, V.E., Uryadov, V.P. and Shumaev V.V., (2003) "Chirp ionosonde and its application in the ionospheric research", Radiophysics and Quantum Electronics, 11(47), 919-952.

12) Ivanov, V.A., Ivanov, D.V. and Ryabova M.I., (2011) "Investigation of the factors that lead to the distortion of high frequency signals with spread spectrum in their quasiantiaircraft propagation in the ionosphere", Electromagnetic Waves and Electronic Systems, 8(16), 33-39.

13) Ivanov, V.A., Ivanov, D.V., Ryabova, M.I. and Lashchevsky A.R., (2008) "Researches of correction the dispersion distortion, that emergents in ionospheric radio channel with passband $1 \mathrm{MHz}$ ", Electromagnetic Waves and Electronic Systems, 8(13), 58-66.

14) Ivanov, V.A., Katkov, E.V. and Chernov A.A., (2010) "Device and algorithms of synchronization of radio engineering communication systems and the sounding of ionospheric high frequency radio channels", Vestnik of Volga State University of Technology. Series "Radio Engineering and Infocommunication Systems", 2, 114-126.

15) Ivanov, V.A., Ryabova, N.V., Tsarev, I.E. and Korkin A.V., (2009) "Automatic determination of the signal-to-noise ratio when measuring the scattering function of narrow-band decameter radio channels", Vestnik of Volga State University of Technology. Series "Radio Engineering and Infocommunication Systems", 3, 3-12.

16) Ivanov, V.A., Ryabova, N.V., Maltcev, A.V. etc., (2014) "Comprehensive methods of vertically-oblique sounding ionograms processing for determining parameters of ionospheric channels", Telecommunications and Radio Engineering, 8, 11-21.

17) Cannon, P.S., Angling, M.J., Davies, N.C. etc., (2000) "DAMSON HF channel characterization - a review", Proc. MILCOM. 59-64.

18) Le Roux, Y. M., Niberon, M., Fleury, R. etc., (1990) "HF channel modeling and simulation", IEE Radio Receivers and Associated Systems $5^{\text {th }}$ conf, Cambridge. 72-76.

19) http://resources.yesican-science.ca/trek/radiation/final/solar flare info.html, date of access 23.07.2015.

20)/http://www.swpc.noaa.gov/NOAAscales/, date of access 23.07.2015.23.07.2015.

Paper sent to revision: 15.10 .2015

Paper ready for publication: 05.12.2016. 